\title{
Upconversion based continuous-wave mid-infrared detection
}

\author{
Tidemand-Lichtenberg, Peter; Dam, Jeppe Seidelin; Pedersen, Christian
}

Published in:

Nonlinear Optics Technical Digest

Publication date:

2013

Document Version

Publisher's PDF, also known as Version of record

Link back to DTU Orbit

Citation (APA):

Tidemand-Lichtenberg, P., Dam, J. S., \& Pedersen, C. (2013). Upconversion based continuous-wave midinfrared detection. In Nonlinear Optics Technical Digest (pp. NM3B.3). Optical Society of America.

\section{General rights}

Copyright and moral rights for the publications made accessible in the public portal are retained by the authors and/or other copyright owners and it is a condition of accessing publications that users recognise and abide by the legal requirements associated with these rights.

- Users may download and print one copy of any publication from the public portal for the purpose of private study or research.

- You may not further distribute the material or use it for any profit-making activity or commercial gain

- You may freely distribute the URL identifying the publication in the public portal

If you believe that this document breaches copyright please contact us providing details, and we will remove access to the work immediately and investigate your claim. 


\title{
Upconversion based continuous-wave mid-infrared detection
}

\author{
Peter Tidemand-Lichtenberg*, Jeppe Seidelin Dam and Christian Pedersen \\ DTU Fotonik, Technical University of Denmark, DK-4000 Roskilde, Denmark \\ ptli@fotonik.dtu.dk
}

\begin{abstract}
We present theoretical and experimental work on upconversion based mid-wavelength infrared detection using silicon detectors without the need for cryogenic cooling. We consider both multi-spectral imaging and point spectroscopy targeting several specific applications.
\end{abstract}

OCIS codes: (190.7220) Upconversion; (300.6340) Spectroscopy, infrared; (100.2960) Image analysis;

\section{Introduction}

Spectroscopy and imaging has for decades been an indispensable method for identification and quantification of chemical compounds in the ultraviolet, visible and near infrared spectral region. However, even though applications have emerged for infrared analysis, field deployable system development has been hampered by the lack of efficient low noise, low cost detectors.

In the early years of nonlinear optics approaches toward image upconversion based on sum frequency mixing was demonstrated [1, 2], allowing for the use of photographic film or other visible detector systems for infrared imaging. However, applications were limited by the low quantum efficiency, particularly for cw systems [3].

In recent years, much progress has been made in the development of microbolometers and low bandgap semiconductor materials such as InSb and MCT detectors. However, these devices are still limited by the need for cryogenic cooling for low noise performance, in strong contrast to visible light detectors.

In this paper we present results on parametric upconversion of infrared radiation into the near visible spectrum for detection using Si-based imaging devices such as CCD cameras. We present results for mid-infrared imaging and spectroscopy, using sum frequency mixing combined with Si-CCD cameras for detection.

\section{Concept and technology}

The concept of the presented approach is to use sum frequency mixing between the infrared signal and a strong intra cavity field in a high finesse diode pumped solid state laser [4]. Note that the conversion efficiency is proportional to the mixing laser power. The incoherent infrared radiation is upconverted in an infinity corrected plane inside the nonlinear media to avoid aberrations caused by the finite thickness of the nonlinear crystal.

The image information from the object is in the back focal plane of a first lens carried as angles, see the left part of Fig. 1. Hence, we have to consider non-collinear interaction in the conversion process, i.e. the phase-matching condition will depend on the spatial position in the object image. The propagation angle of the upconverted radiation is further scaled according to the ratio of the input to the output wavelength.

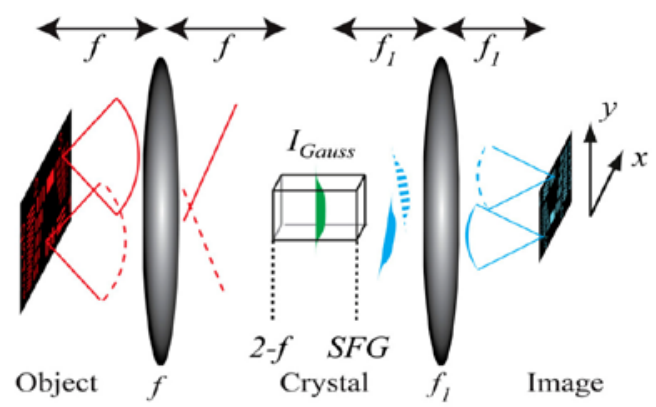

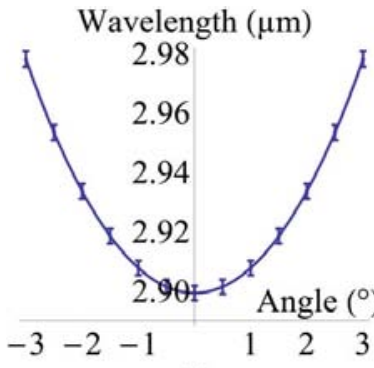

(a)

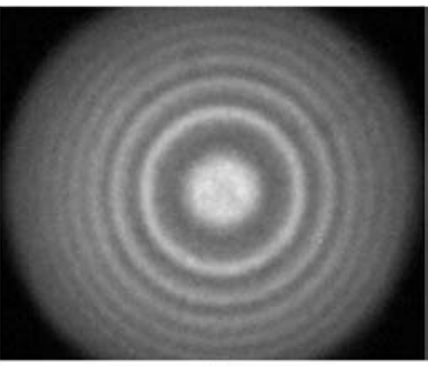

(b)

Fig. 1. Left: An object is emitting incoherent light, which can be modeled as point sources emitting spherical waves. A lens, $f$, transforms these spherical waves to plane waves. The plane waves are cropped by the on-axis Gaussian mixing beam and shifted to a different wavelength. These waves exit the non-linear crystal at a smaller angle due to momentum conservation. The individually upconverted Gaussian waves forms an image after a second lens, $f 1$, in the image plane. Right: (a) Phase-match wavelength as a function of propagation angle through the nonlinear crystal. Error bars indicates phase-matched bandwidth. (b) The upconverted image of mid-IR emission from hot water vapor around $2.9 \mu \mathrm{m}$. 


\section{Imaging, spectroscopy and multispectral imaging}

Configuring the system as "infinity corrected" makes it quite similar to a pin-hole camera. However, the upconversion system also shifts the wavelength of the entire infrared image to a spectral region where detector technology has been most developed. The spatial resolution of the system is in the infinity corrected configuration is set by the beam size of the mixing laser field, which acts as a soft aperture of the system. Depending on the nature of the illumination being spatially coherent or incoherent the appropriate transfer function can be found in terms of a point spread function, i.e. the Fourier transform of the Gaussian mixing laser beam [5].

In addition to the spatial filtering caused by the mixing laser field, the upconverted field depends on the spectral acceptance parameters of the nonlinear conversion process. Depending on the specific application, the phasematching properties can be designed to be broadband, narrowband or even tunable. But using a large field of view an angular spectral dependence in the image conversion is unavoidable.

It is possible to use the spectral curvature of the upconverted images to design a simple spectrometer for infrared measurements. Assuming an infrared signal with spectral signatures, it is possible to exploit the relation between phase-match angle and internal propagation angle of the upconverted infrared light to measure the spectral content in a very simple manner, see Fig. 1, (a). The right part of Fig. 1, (b) shows an upconverted image of the emission from hot water vapor at $2.9 \mu \mathrm{m}$. Very distinct concentric rings are seen in the image. Analyzing the image using the spectral response from (a) it is straightforward to calculate the emission spectrum from the hot water vapor. Based on an extension of this approach we have reached a spectral resolution down to $0.2 \mathrm{~nm}$ at $2.9 \mu \mathrm{m}$ [6].

Many interesting chemical compounds have distinct spectral features in the mid-infrared spectral region. It is therefore desirable to make multispectral images in this region with sufficient spectral resolution to differentiate chemical substances and their spatial distribution. We present an approach to generate multiple monochromatic images based on phase-match scanning of upconverted images.

\section{Conclusion}

Based on continuous wave image upconversion, we present an efficient low noise approach for infrared imaging and spectroscopy. This approach has significant advantages in terms of noise and cost efficiency in applications where detailed spectral infrared features are important. We present various configurations and there applicability to specific applications.

\section{References}

[1] J. E. Midwinter and J. Warner, “Up-Conversion of Near Infrared to Visible Radiation in Lithium-meta-Niobate*, J. Appl. Phys. 38, 519 (1967).

[2] L. Gampel and F. M. Johnson, “IR Image Detection by CW Parametric Up-Conversion to the Visible,” IEEE J. Quantum Electron. Conference, 354 (1968).

[3] R. A. Andrews, “IR image parametric up-conversion, “ IEEE J. Quantum Electron. QE6, 68-80 (1970).

[4] J. S. Dam, P. Tidemand-Lichtenberg and C. Pedersen, "Room-temperature mid-infrared single-photon spectral imaging, "Nature Photonics, 6, 788-793 (2012).

[5] J. S. Dam, C. Pedersen and P. Tidemand-Lichtenberg, “Theory for upconversion of incoherent images, “ Opt. Express, 20, 1475-1482 (2012).

[6] Q. Hu, J. S. Dam, C. Pedersen and P. Tidemand-Lichtenberg, "High-resolution mid-IR spectrometer based on frequency upconversion, “ Opt. Lett., 37, 5232-5234 (2012). 